\title{
Some applications of the generalised Poisson-Jensen formula
}

\author{
By S. A. ScotT.
}

(Received 12th December, 1939. Read 3rd February, 1940.)

§1. The object of this paper is to show how some formulae in Analytic Number Theory, in particular, the formula for $N(T)$, the number of zeros of the Zeta-function between $t=0$ and $t=T$, are easy deductions from the Generalised Poisson-Jensen formula. A similar method, using Green's function instead of the general function $g(s)$ of $\S 2$, has been published by F. and R. Nevanlinna (Math. Zeitschrift, 20 (1924), and 23 (1925), but the result contained in (vi) below appears to be new, although the writer has not been able, as yet, to make any effective use of it. It is clear that other applications could be made, but it seems sufficient to give here an indication of the method. The notation throughout is the usual one, and the references are to the Cambridge Tract by E. C. Titchmarsh on "The Zeta-function of Riemann." Finally, I am indebted to the referee for the reference to the papers of $F$. and R. Nevanlinna.

$\S 2$. Suppose that $f(s)$ is meromorphic with zeros $a_{\mu}$ and poles $b_{\nu}(\mu, \nu=1,2,3 \ldots)$ in the domain $G$, and no zeros or poles on the boundary $\Gamma$ of this domain. Let $g(s)$ be a real function of $s=\sigma+t i$ having continuous partial derivatives up to the second order in $G$, and denote the area-element of $G$ by $d \Sigma$ and the arc-element of $\Gamma$ by $d u$. Then we have the well-known Generalised Poisson-Jensen formula:

$$
\begin{aligned}
-2 \pi \sum_{\mu} g\left(a_{\mu}\right)+2 \pi \Sigma_{\nu} g\left(b_{\nu}\right) & =\iint_{G} \log |f(s)| \Delta g(s) d \Sigma \\
& +\int_{\Gamma}\left\{\log |f(s)| \frac{\partial g(s)}{\partial n}-g(s) \frac{\partial}{\partial n} \log |f(s)|\right\} d u .
\end{aligned}
$$

Here the derivative $\frac{\partial}{\partial n}$ is taken along the inward normal, and $\Delta$ 
stands for the Laplacian operator $\left(\frac{\partial^{2}}{\partial \sigma^{2}}+\frac{\partial^{2}}{\partial t^{2}}\right)$. In what follows, we take $f(s)=\zeta(s)$, Riemann's Zeta-function, and consider the results of giving $g(s)$ various special values, remembering the symmetry of the zeros of $\zeta(s)$ about the line $\sigma=\frac{1}{2} . \quad G$ is taken to be an infinite rectangle in each application. In case there are zeros (or poles) on $\Gamma$ (as happens in the applications (iv), ( $v$ ) and (vi) below), the above formula must be slightly modified. The terms $g\left(a_{\mu}\right)$ (or $g\left(b_{v}\right)$ ) corresponding to these zeros (or poles) must be multiplied by $\frac{1}{2}$, and the line integral on the right is an improper one and is defined in the usual manner.

§3. (i) $g(s)=\sigma-\frac{1}{2}, G$ being the region $0 \leqq \sigma \leqq \infty ; 2 \leqq t \leqq T$.

We suppose that $T$ is not the ordinate of a zero of $\zeta(s)$.

Here we have, since $\Delta g(s)=0$,

$$
\begin{aligned}
& 0=-2 \sum_{\mu}\left(\sigma_{\mu}-\frac{1}{2}\right)=\int_{0}^{\infty}\left(\frac{1}{2}-\sigma\right)\left[\frac{\partial}{\partial t} \log |\zeta(\sigma+t i)|\right]_{t=2} d \sigma \\
& -\int_{\infty}^{0}\left(\sigma-\frac{1}{2}\right)\left[-\frac{\partial}{\partial t} \log |\zeta(\sigma+t i)|\right]_{t=T} d \sigma+\int_{R}^{2}\left\{\log |\zeta(t i)|+\frac{1}{2}\left[\frac{\partial}{\partial \sigma} \log |\zeta(\sigma+i i)|\right]_{\sigma=0}\right\} d t,
\end{aligned}
$$

and, on using the Cauchy-Riemann equations, this becomes

$$
\begin{aligned}
0= & \int_{0}^{\infty}\left\{\left(\sigma-\frac{1}{2}\right) \frac{\partial}{\partial \sigma} \arg \zeta(\sigma+2 i)\right\} d \sigma+\int_{0}^{\infty}\left\{\left(\sigma-\frac{1}{2}\right) \frac{\partial}{\partial \sigma} \arg \zeta(\sigma+T i)\right\} d \sigma \\
& -\int_{2}^{T} \log |\zeta(t i)| d t-\frac{1}{2} \int_{2}^{T}\left\{\frac{\partial}{\partial t} \arg \zeta(t i)\right\} d t \\
= & {\left[\left(\sigma-\frac{1}{2}\right)\{\arg \zeta(\sigma+2 i)+\arg \zeta(\sigma+T i)\}\right]_{0}^{\infty}-\int_{0}^{\infty} \arg \zeta(\sigma+2 i) d \sigma } \\
& -\int_{0}^{\infty} \arg \zeta(\sigma+T i) d \sigma-\int_{2}^{T} \log |\zeta(t i)| d t-\frac{1}{2} \arg \zeta(T i)+\frac{1}{2} \arg \zeta(2 i) \\
= & -\int_{2}^{T} \log \zeta(t i) \mid d t-\int_{0}^{\infty} \arg \zeta(\sigma+T i) d \sigma+O(1),
\end{aligned}
$$

(where the $O(1)$ is an absolute constant), since $\arg \zeta(s)=O\left(e^{-A \sigma}\right)$ for $\sigma>2$.

(ii) $g(s)=\sigma$, G being the region $1 \leqq \sigma \leqq \infty ; 2 \leqq t \leqq T$. 
Here the same method gives

$$
0=\int_{2}^{T} \log |\zeta(1+t i)| d t+\int_{1}^{\infty} \arg \zeta(\sigma+T i) d \sigma+O(1) .
$$

(iii) $g(s)=\sigma ; G$ being the region $0 \leqq \sigma \leqq \infty ; 2 \leqq t \leqq T$.

Here, and in the sequel, we suppose $\zeta(\sigma+T i) \neq 0$ for any $\sigma$.

We have

$$
\begin{aligned}
2 \pi \underset{\mu}{\Sigma} \sigma_{\mu} & =\pi N(T)=\int_{2}^{T} \log |\zeta(t i)| d t+\int_{0}^{\infty} \arg \zeta(\sigma+T i) d \sigma+O(1) \\
& =\int_{2}^{T} \log |\chi(1-t i)| d t+\int_{2}^{T} \log |\zeta(1-t i)| d t+\int_{0}^{\infty} \arg \zeta(\sigma+T i) d \sigma+O(1),
\end{aligned}
$$

where

$$
\chi(s)=2(2 \pi)^{-s} \cos \frac{1}{2} \pi s \Gamma(s)=\frac{\zeta(1-s)}{\zeta(s)} .
$$

Hence we obtain, with the aid of (ii) and Stirling's Theorem,

$$
\pi N(T)=\frac{1}{2} T \log T-\frac{1}{2} T(1+\log 2 \pi)+\int_{0}^{1} \arg \zeta(\sigma+T i) d \sigma+O(1) .
$$

Since $\arg \zeta(s)=O(\log T)$ in the range of the integral, the wellknown formula for $N(T)$ follows, namely

$$
N(T)=\frac{T}{2 \pi} \log \frac{T}{2 \pi}-\frac{T}{2 \pi}+O(\log T) .
$$

§4. (iv) $g(s)=\sigma-\sigma_{0},\left(\frac{1}{2}>\sigma_{0}>0\right), G$ being the region $\sigma_{0} \leqq \sigma \leqq \infty$; $2 \leqq t \leqq T$.

We find

$2 \pi \sum_{\sigma_{\mu} \geqq \sigma_{o}}\left(\sigma_{\mu}-\sigma_{0}\right)=\int_{2}^{T} \log \left|\zeta\left(\sigma_{0}+t i\right)\right| d t+\int_{\sigma_{o}}^{\infty} \arg \zeta(\sigma+T i) d \sigma-\int_{\sigma_{o}}^{\infty} \arg \zeta(\sigma+2 i) d \sigma$ Write $\sigma_{0}=\frac{1}{2}-\kappa$ where $0<\kappa<\frac{1}{2}$, and, with the usual notation, let $N\left(\sigma^{\prime}, T^{\prime}\right)$ denote the number of zeros $\rho$ of $\zeta(s)$ with $\mathscr{R}(\rho)>\sigma^{\prime}$ and $0<\mathscr{O}(\rho)<T$.

Write

and

$$
\int_{\sigma^{\prime}}^{1} N(\sigma, T) d \sigma=N^{*}\left(\sigma^{\prime}, T\right)
$$

$$
N\left(\sigma_{1}, T\right)-N\left(\sigma_{2}, T\right)=N\left(\sigma_{1}, \sigma_{2}, T\right) .
$$


Then we have, using a Stieltjes' Integral,

$$
\begin{aligned}
& 2 \pi \underset{\sigma_{\mu} \geqq \sigma_{0}}{\sum_{\mu}}\left(\sigma_{\mu}-\sigma_{0}\right)=2 \pi \kappa N\left(\frac{1}{2}-\kappa, \frac{1}{2}+\kappa ; T\right)+2 \pi \int_{t+\kappa}^{1}\left(\sigma-\frac{1}{2}+\kappa\right)\{-d N(\sigma, T)\} \\
& =2 \pi \kappa N\left(\frac{1}{2}-\kappa, \frac{1}{2}+\kappa ; T\right)+4 \pi \kappa N\left(\frac{1}{2}+\kappa, T\right)+2 \pi N^{*}\left(\frac{1}{2}+\kappa, T\right) . \\
& =2 \pi \kappa N^{*}(T)+2 \pi N^{*}\left(\frac{1}{2}+\kappa, T\right) \text {. }
\end{aligned}
$$

Hence, finally, we find

$$
\begin{aligned}
& 2 \pi \kappa N(T)+2 \pi N^{*}\left(\frac{1}{2}+\kappa, T\right)=\int_{2}^{T} \log \left|\zeta\left(\frac{1}{2}+\kappa+t i\right)\right| d t \\
& \quad+\int_{2}^{T} \log \left|\chi\left(\frac{1}{2}+\kappa+t i\right)\right| d t+\int_{\frac{1}{2}-\kappa}^{\infty} \arg \zeta(\sigma+T i) d \sigma-\int_{\frac{1}{t-\kappa}}^{\infty} \arg \zeta(\sigma+2 i) d \sigma .
\end{aligned}
$$

It is easy to see that the result also holds for $\kappa=0$.

(v) $g(s)=\sigma-\frac{1}{2}-\kappa,\left(0<\kappa<\frac{1}{2}\right), G$ being the region $\frac{1}{2}+\kappa \leqq \sigma \leqq \infty ; 2 \leqq t \leqq T$.

Here we find

$$
2 \pi N^{*}\left(\frac{1}{2}+\kappa, T\right)=\int_{2}^{T} \log \left|\zeta\left(\frac{1}{2}+\kappa+t i\right)\right| d t+\int_{\frac{b}{2+\kappa}}^{\infty} \arg \zeta(\sigma+T i) d \sigma-\int_{\frac{i}{2}+\kappa}^{\infty} \arg \zeta(\sigma+2 i) d \sigma .
$$

From (iv) and (v) we deduce

$$
\begin{aligned}
& 2 \pi \kappa N(T)=\int_{2}^{T} \log \left|\chi\left(\frac{1}{2}+\kappa+t i\right)\right| d t+\int_{\frac{1}{2}-\kappa}^{t+\kappa} \arg \zeta(\sigma+T i) d \sigma \\
& \quad-\int_{i-\kappa}^{1+\kappa} \arg \zeta(\sigma+2 i) d \sigma .
\end{aligned}
$$

§5. (vi) $g(s)=\log |\chi(s)|, G$ being the region $\frac{1}{2} \leqq \sigma \leqq \infty ; 2 \leqq t \leqq T$.

Here we find, since $g(s)$ is harmonic,

$$
\begin{aligned}
& \sum_{\beta_{\mu} \geqq \frac{1}{2}} \log \left|\chi\left(\rho_{\mu}\right)\right|=\int_{2}^{T} \log \left|\zeta\left(\frac{1}{2}+t i\right)\right| \mathscr{R}\left\{\frac{\chi^{\prime}}{\chi}\left(\frac{1}{2}+t i\right)\right\} d t \\
& \quad+\int_{\frac{1}{\underline{Z}}}^{\infty} \log : \zeta(\sigma+T i)\left|\mathcal{O}\left\{\frac{\chi^{\prime}}{\chi}(\sigma+T i)\right\} d \sigma-\int_{\frac{2}{\sharp}}^{x} \log \right| \chi(\sigma+T i) \mid \mathscr{O}\left\{\frac{\zeta^{\prime}}{\zeta}(\sigma+T i)\right\} d \sigma+O(1),
\end{aligned}
$$

where $\rho_{\mu}=\beta_{\mu}+\gamma_{\mu} i\left(\gamma_{\mu}>0\right)$ is a typical zero of $\zeta(s)$, and the summation on the left is over all zeros for which $\beta_{\mu} \geqq \frac{1}{2}, 2<\gamma_{\mu}<T$.

This sum is easily seen to be asymptotically equal to

$$
\sum_{\beta_{\mu} \geqq !}\left(\beta_{\mu}-\frac{1}{2}\right) \log \gamma_{\mu} .
$$


The right-hand side is equal to

$$
\begin{aligned}
& \int_{2}^{T} \log \mid \zeta\left(\frac{1}{2}+t i\right) !\left\{-\mathcal{Q}\left(\frac{\zeta^{\prime}}{\zeta}\left(\frac{1}{2}-t i\right)\right)-\mathcal{Q}\left(\frac{\zeta^{\prime}}{\zeta}\left(\frac{1}{2}+t i\right)\right)\right\} d t+I_{1}-I_{2}+O(1) \\
& =-2 \int_{2}^{T} \log \mid \zeta\left(\frac{1}{2}+t i\right)^{\prime} \mathcal{O}\left(\frac{\zeta^{\prime}}{\zeta}\left(\frac{1}{2}+t i\right)\right) d t+I_{1}-I_{2}+O(1),
\end{aligned}
$$

where

$$
\begin{aligned}
I_{1}-I_{2}= & -\int_{\vdots}^{\infty} \log |\zeta(\sigma+T i)| \mathcal{g}\left(\frac{\zeta^{\prime}}{\zeta}(1-\sigma-T i)\right) d \sigma \\
& -\int_{i}^{\infty} \log |\zeta(1-\sigma-T i)| \mathcal{g}\left(\frac{\zeta^{\prime}}{\zeta}(\sigma+T i)\right) d \sigma \\
= & -X-Y .
\end{aligned}
$$

It is known (Titchmarsh: The Zeta-function of Riemann, p. 61) that $\frac{\zeta^{\prime}}{\zeta}(s)=O(\log |s|)$ for $\sigma \leqq-1, t \geqq t_{0} ;$ and also that $|\zeta(s) j|=1+O\left(e^{-\Delta \sigma}\right)$ for $\sigma>2$. It follows that in the term $X$ above, $\int_{2}^{\infty}$ is $O(\log T)$. In the term $Y$, for $\sigma \geqq 2$ we have

$$
\log |\zeta(1-\sigma-T i)|=O\left(\frac{\sigma}{2} \log \left(\sigma^{2}+T^{2}\right)\right)
$$

by the functional equation; and we may replace $\mathcal{O}\left(\frac{\zeta^{\prime}}{\zeta}(\sigma+T i)\right)$ by a uniformly convergent Dirichlet's series and then integrate termwise. A slight calculation then shows that, in $Y$ also, $\int_{2}^{\infty}$ is $O(\log T)$.

We have now to discuss the residual part

$$
Z=-\int_{:}^{2} \log |\zeta(\sigma+T i)| \mathcal{g}\left(\frac{\zeta^{\prime}}{\zeta}(1-\sigma-T i)\right) d \sigma-\int_{:}^{2} \log |\zeta(1-\sigma-T i)| \mathcal{J}\left(\frac{\zeta^{\prime}}{\zeta}(\sigma+T i)\right) d \sigma .
$$

Now we have (Titchmarsh, ibid., p. 27)

$$
\frac{\zeta^{\prime}}{\zeta}(s)=\underset{\mid T-\gamma_{i}<1}{\Sigma} \frac{1}{s-\rho}+O(\log T)
$$

uniformly in $-1 \leqq \sigma \leqq 2$, where $\rho$ is a typical zero of $\zeta(s)$. 
It is also known that

$$
N(T+1)-N(T)=O(\log T),
$$

and that $\zeta(s)=O\left(T^{\frac{1}{2}}\right)$ for $s=\sigma+T i, \frac{1}{2} \leqq \sigma \leqq 1$. Using these results, it is easy to see that $Z=O\left(\log ^{2} T\right)$.

We thus have, finally,

$\underset{\beta_{\mu} \geqq \frac{1}{2}}{\sum_{2}} \log \left|\chi\left(\rho_{\mu}\right)\right|=-2 \int_{2}^{T} \log \left|\zeta\left(\frac{1}{2}+t i\right)\right| \mathscr{R}\left\{\frac{\zeta^{\prime}}{\zeta}\left(\frac{1}{2}+t i\right)\right\} d t+O\left(\log ^{2} T\right)$.

\section{Trinity College,}

DUbLin. 